In der Rubrik „Literatur kompakt" werden die wichtigsten Originalarbeiten aus der internationalen Fachliteratur referiert.

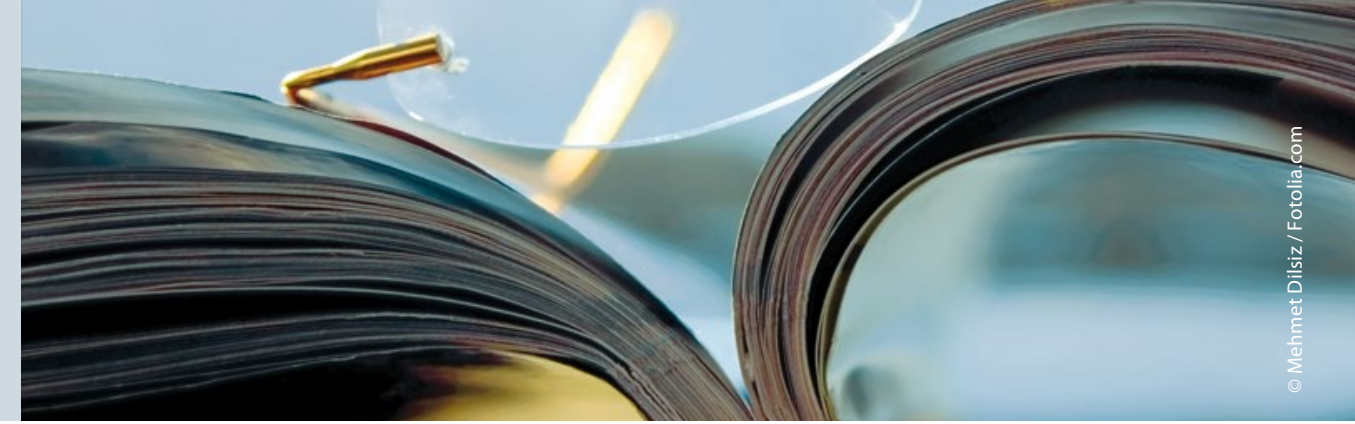

\section{Erneut erhöhte Blasenkrebsrate unter Pioglitazon}

\section{Bei Diabetikern unter Pioglitazon ist das Risiko für Blasentumoren um etwa $60 \%$ erhöht. Mit Rosiglitazon lässt sich dagegen keine gesteigerte Blasen-} krebsinzidenz feststellen. Dies deutet auf ein substanzspezifisches Risiko hin.

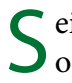
eit der PROACTIVE(Prospective Pioglitazone Clinical Trial in Macrovascular Events)-Studie aus dem Jahr 2005 wird über ein erhöhtes Blasenkrebsrisiko unter Pioglitazon diskutiert. Damals sind bei Diabetikern mit dem Glitazon im Vergleich zur Placebogruppe gehäuft Blasentumoren aufgetreten. $\mathrm{Zu}$ ähnlichen Ergebnissen kamen einige Observationsstudien, in anderen wurde dagegen keine erhöhte Rate des Tumors unter Pioglitazon festgestellt. Eine große Kohortenanalyse von fast 150.000 Diabetikern, die im Schnitt knapp fünf Jahre lang untersucht worden waren, scheint nun die ursprüngliche Befürchtung zu bestätigen.

Für die Studie haben Forscher um Dr. Marco Tuccori vom Jewish General Hospital in Montreal, Kanada, das große britische Patientenregister „Clinical Practice Research Datalink“ (CPRD) durchforstet. Das Team um Tuccori interessierte sich ausschließlich für Patienten, die zwischen 2000 und 2013 entweder erstmals antidiabetisch behandelt oder in dieser Zeit auf ein neues Antidiabetikum eingestellt worden waren. Die Forscher nahmen ferner nur Patienten über 40 Jahre in die Analyse auf, die schon mindestens ein Jahr in der Datenbank registriert waren. Insgesamt kamen auf diese Weise rund 690.000 Patientenjahre zusammen. Dabei entwickelten 622 Diabetiker einen bösartigen Blasentumor, was einer Inzidenz von rund $90 \mathrm{Tu}$ - moren auf 100.000 Patientenjahre entspricht. Im Schnitt traten die Tumoren 4,4 Jahre nach Aufnahme in die Studie auf. Insgesamt hatten nur 921 Patienten Pioglitazon bekommen. Bei 54 von ihnen entwickelte sich ein Blasentumor. Das entspricht einer Inzidenz von 122 Tumoren pro 100.000 Patientenjahre, bei den Diabetikern ohne Glitazone waren es nur 89 pro 100.000 .

Unter Berücksichtigung von Risiken und Begleitfaktoren wie Alter, BMI, Dauer des Typ-2-Diabetes und Blasenkrebsrisikofaktoren wie Zystitis, Blasensteine, Rauchen und Alkoholismus ließ sich daraus eine um $63 \%$ erhöhte Tumorrate errechnen - verglichen mit $\mathrm{Pa}$ tienten, die andere Medikamente als Glitazone zur Diabetesbehandlung erhalten hatten. Die Inzidenz stieg mit der Dauer der Therapie und mit der Gesamtdosis. Etwas mehr als 2.100 Diabetiker waren mit Rosiglitazon behandelt worden. Für diese Patienten ließ sich kein signifikant erhöhtes Blasenkrebsrisiko nachweisen - die Inzidenz lag bei 86 Tumoren pro 100.000 Patientenjahre. Auch zeigte sich kein Zusammenhang zwischen der Blasenkrebsinzidenz und Therapiedauer oder Gesamtdosis.

Die Forscher um Tuccori schauten als analysen, wie robust die Ergebnisse waren. Mal schlossen sie Patienten mit Tumordiagnosen in den ersten beiden Studienjahren aus, da die Krebsentwicklung nächstes anhand von neun Sensitivitäts- hier wohl eher nicht nennenswert durch die Medikation beeinflusst wurde, mal berücksichtigten sie nur Patienten mit einer gewissen Glitazon-Mindestdosis, ein anderes Mal schlossen sie Patienten aus, bei denen schon vor dem Studienbeginn eine Reihe von Risikofaktoren oder Blasenproblemen vorgelegen hatten - stets zeigte sich ein signifikant erhöhtes Risiko für Pioglitazon (zwischen 46 und 76 $\%)$, nicht aber für Rosiglitazon.

Fazit: Da sich anhand der Daten sowohl für die Therapiedauer als auch für die Dosis eine deutliche Erhöhung des Krebsrisikos unter Pioglitazon, nicht aber Rosiglitazon berechnen lässt, halten die Studienautoren einen substanzspezifischen und weniger einen klassenspezifischen Effekt für wahrscheinlich. Da die Resultate der CPRD-Analyse auf nur 54 Blasentumoren unter Pioglitazon beruhen, kann der Zufall das Ergebnis deutlich verzerrt haben.

Das Bundesinstitut für Arzneimittel und Medizinprodukte (BfArM) hat den Hersteller von Pioglitazon bereits 2011 zu einem Rote-Hand-Brief veranlasst, in dem auf das Blasenkrebsrisiko hingewiesen wird. Das Institut empfahl Ärzten zudem, keine neuen Patienten mehr auf Pioglitazon einzustellen. Die europäische Zulassungsbehörde EMA sieht nach wie vor einen überwiegenden Nutzen der Arznei zur Second- und Thirdline-Therapie, wenn eine Behandlung mit Metformin oder anderen Antidiabetika nicht möglich ist.

Thomas Müller

Tuccori M, Filion KB, Yin $\mathrm{H}$ et al. Pioglitazone use and risk of bladder cancer: population based cohort study. BMJ. 2016;352:i1541. 